\section{Rotaviren beim Pferd}

\section{P. A. Bachmann, H. Werbahn, R. Jacobi und W. Eichborn}

Institut für Medizinische Mikrobiologie, Infektions- und Seuchenmedizin, Tierärztliche Fakultät, Universität München, Vorstand: Prof. A. Mayr, und Tierklinik Sottrum

Rotavirusinfektionen verursachen Diarrhöen bei Neugeborenen zahlreicher Spezies. Sie kommen auch bei Fohlen vor. Diese Infektionen sind streng auf den Intestinaltrakt begrenzt und charakterisiert durch akuten Durchfall mit wäßrigen, gelblichen Faeces, Niedergeschlagenheit und Schwäche. Infizierte, differenzierte Dünndarmepithelzellen verlieren ihre unterschiedlichen Funktionen, lösen sich $a b$ und werden größtenteils zerstört. Die Folge ist ein Malabsorptionssyndrom mit Durchfall, Dehydratation und in seltenen Fällen - letalem Ausgang.

Rotavirusinfektionen bei Fohlen werden häufig begleitet von Infektionen mit anderen enteropathogenen Erregern wie Salmonellen, E. coli, Campylobacter sp. und/oder Coronaviren (Eugster et al., 1978; Durbam et al., 1979; Conner und Darlington, 1980; Tzipori et al., 1982). Sie sind bei neugeborenen Fohlen mit unterschiedlicher Schwere der Symptome häufig anzutreffen.

Equine Rotaviren werden dem Genus Rotavirus innerhalb der Familie Reoviridae zugeordnet. Sie sind 65 bis $75 \mathrm{~nm}$ im Durchmesser, ikosaederförmig und tragen eine doppelte Kapsomerenschale (Abb. 1). Das Genom der Virionen besteht aus doppelsträngiger RNS, die in 11 einzelnen Segmenten vorliegt (Tzipori et al., 1979; Rodger et al., 1980). Das Wanderungsverhalten der RNS-Segmente equiner Rotaviren entspricht denen von anderen Säugetieren der Gruppe A, zeigt jedoch ein deutliches Speziesmuster. Die RNS-Segmente kodieren für 10-11 virusspezifische Proteine, von denen vier im Inneren des Virions und vier in der äußeren Kapsidhülle lokalisiert sind (Gillespie et al., 1984). Die Rotaviren der Pferde haben ein inneres Viriongruppenantigen gemeinsam mit anderen Säugerrotaviren der Gruppe A, das vor allem für die Virusdiagnostik von großem Wert ist. Es läßt sich im Enzyme-linked Immunosorbent Assay (ELISA), in der Komplementbindungsreaktion, in der Immunodiffusion und mit Hilfe der Immunofluoreszenztechnik nachweisen (Thouless et al., 1977). Im Neutralisationstest sind bisher zwei unterschiedliche Serotypen differenziert worden (Hoshino et al., 1983 b). Darüber hinaus besitzt der equine $\mathrm{H}$-1-Virusstamm hämagglutinierende Aktivitäten für Erythrozyten vom Menschen, Meerschweinchen und von Affen, während der equine Stamm $\mathrm{H}-2$ nicht hämagglutiniert.

Einige Stämme von equinen Rotaviren können in der Rhesusaffennieren-Zellinie Ma-104 in vitro mit einem cytopathischen Effekt gezüchtet werden (Hoshino et al., 1983 a; Gillespie et al., 1984; Eichborn et al., 1985).

\section{Zusammenfassung}

In einer Übersicht wird der heutige Kenntnisstand der equinen Rotavirusinfektion unter besonderer Berücksichtigung der Pathogenese, Epizootiologie und Immunologie dargestellt. Rotavirusinfektionen sind beim Pferd endemisch und können allein oder im Zusammenspiel mit bakteriellen Erregern schwerste Diarrhöen auch mit Todesfolge hervorrufen. Eine wirksame Prophylaxe steht derzeit nicht zur Verfügung. Eigene Erfahrungen auf den Gebieten Klinik und Diagnostik der Rotavirusinfektion beim Fohlen werden berichtet.

\section{Equine Rotavirus Infections}

Present knowledge on equine rotaviral infection is reviewed with special emphasis on pathogenesis, epizootiology and immunology. Rotavirus infections in the horse are endemic and can - alone or in combination with bacterial agents - induce most severe diarrheas also with lethal outcome. Efficient prophylactic measures are presently not available. Own experiences in the fields of clinical symptoms and diagnosis of rotavirus infections in foals are reported.

Rotaviren sind unter natürlichen Verhältnissen sehr stabil. Sie können in Ställen auch in getrocknetem Zustand monatelang infektiös bleiben. Für die Desinfektion eignen sich Äthanol (70 \%), Lysol (5\%) und Hexachlorophenverbindungen (Snodgrass und Herring, 1977; Sattar, 1983). Iodophore, quaternäre Ammoniumverbindungen, Natriumhypochlorit und Chlor in Konzentrationen, die Trinkwasser zugesetzt werden, sind unwirksam (Sattar, 1983).

Das Vorkommen von Rotavirusinfektionen bei Pferden ist bisher in den USA (Conner und Darlington, 1980), Australien (Studdert et al., 1978; Tzipori und Walker, 1979), Neuseeland (Durbam et al., 1979), Japan (Imagawa et al., 1982), Großbritannien (Flewett et al., 1975), Irland (Strickland et al., 1982), Frankreich (Conner und Darlington, 1980) und der Bundesrepublik Deutschland (Eichhorn et al., 1985) beschrieben worden. Wahrscheinlich ist der Erreger weltweit verbreitet. Krankheitsausbrüche sind in Gestüten mit großen Fohlenzahlen häufig (Tzipori und Walker, 1978; Strick. land et al., 1982; Eichborn et al., 1985). Die Infektion breitet sich bei empfänglichen Fohlen im Alter zwischen 4 bis 5 Tagen und 3 bis 4 Monaten rasch aus. Da die Abfohlzeiten in Gestüten sich auf wenige Wochen oder Monate im Jahr konzentrieren, ist anzunehmen, daß ein wichtiger Faktor in der Epizootiologie der Erkrankungen im hohen Infektionsdruck liegt, der sich während der Abfohlsaison aufbaut.

Wie bei anderen Spezies (Bachmann und Hess, 1982) sind Rotavirusinfektionen beim Fohlen im Dünndarm lokalisiert (Conner und Darlington, 1980). Das Virus wird oral aufgenommen und vermehrt sich hauptsächlich im Dünndarm. Eine Ausbreitung des Virus über die Dünndarmepithelzellen hinaus oder eine Virämie werden nicht beobachtet. Die pathologischen Veränderungen bestehen in mononukleären Zellinfiltrationen und Atrophie der Villi. Ultrahistologisch lassen sich ein Verlust der Mikrovilli, eine Kernwandhyperchromasie und Vakuolisierung in infizierten Zellen nachweisen (Conner und Darlington, 1980). Pferderotaviren werden mit den Faeces ausgeschieden, wo sie während der akuten Erkrankung für ungefähr 5 bis 9 
Tage nachgewiesen werden können. Rotaviren werden jedoch auch von gesunden Pferden ausgeschieden. Nach primärer Infektion kann Rotavirus intermittierend bis zu 6 bis 8 Monaten im Kot dieser Tiere nachgewiesen werden (Conner et al., 1983).

Nach einer Inkubationszeit von 1 bis 3 Tagen treten Durchfallerscheinungen plötzlich und ohne Vorankündigung auf. $\mathrm{Zu}$ Beginn der Erkrankungen kann es zu einer Erhöhung der Körpertemperatur auf $39,5^{\circ} \mathrm{C}$, in Ausnah. mefällen auf $40-41^{\circ} \mathrm{C}$, kommen. Der Kot ist wäßrig bis cremig, gelblich mit einem stinkenden Geruch. In der Regel ändert sich die Farbe am 2. Tag nach Beginn der Erscheinungen. Der Kot wird grau und sieht aus wie „dünnflüssiger Beton". Erkrankte Fohlen sind in ihrem Allgemeinbefinden gestört, sie sind teilnahmslos und lethargisch. In schweren Fällen tritt Schwäche ein, und die Fohlen saugen nicht mehr an der Mutter. Ihr Allgemeinzustand verschlechtert sich rapide mit zunehmender Dauer der Erkrankung. Bei allen Erkrankten läßt sich eine deutliche Exsikkose diagnostizieren, mit mattem, struppigem Fell. Bei einigen Ausbrüchen ist neben hoher Morbidität auch eine hohe Mortalität beobachtet worden. Die Dauer der Diarrhö kann zwischen 2 und 12 Tagen betragen. Junge Fohlen entwickeln schwerere Symptome als ältere Tiere (Eugster et al., 1978). Bei milden Fällen dauert der Durchfall nur etwa 24 bis 48 Stunden mit schneller Rekonvaleszenz.

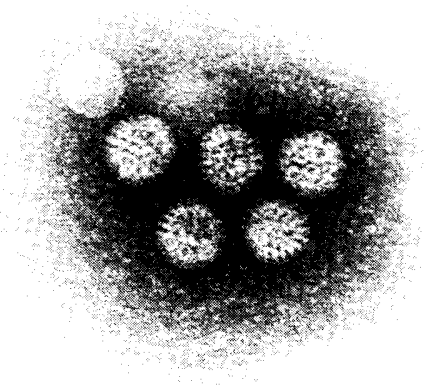

Abb. 1: Elektronenmikroskopische Aufnahme von Rotaviren im Kot eines an Diarrhö erkrankten Fohlens.

Die Diagnose der Rotavirusinfektion beim Pferd wird durch den Virus- bzw. Antigennachweis in Faecesproben vorgenommen. $\mathrm{Da}$ eine Routineisolierung der Pferderotaviren in Zellkulturen schwierig ist, werden vor allem der ELISA und die Immunelektronenmikroskopie (IEM) eingesetzt. Mit beiden Verfahren ist eine Diagnose in wenigen Stunden möglich. Am besten geeignet ist der ELISA, mit dem ein gruppenspezifisches Antigen bei Pferderotaviren nachgewiesen werden kann. Der ELISA wird von uns seit einigen Jahren routinemäßig bei der Rotavirusdiagnose der Haustiere inklusive des Pferdes eingesetzt (Bacbmann,
1979). Der Nachweis von Serumantikörpern ist weniger geeignet, da die meisten Pferde Antikörper gegen Rotavirus haben und der Nachweis von Serumantikörpern keinen Aufschluß über akute Infektionen oder über die lokale Immunitätslage gibt. Infektionen sind weit verbreitet (Conner und Darlington, 1980; Imagawa et al., 1982; Tzipori et al., 1982).

Prophylaktische Maßnahmen stehen derzeit nicht zur Verfügung. Erkrankte Fohlen werden symptomatisch oral oder parenteral mit Elektrolytersatz behandelt (Studdert et al., 1978; Strickland et al., 1982). Weiterhin wird eine systematische Antibiotikabehandlung über 3 bis 5 Tage empfohlen, verbunden mit der Gabe von Spasmolytika. Erkrankte Fohlen können mit Magermilch-Joghurt gefüttert werden. Zur Vorbeuge können Kolostrumgemelke, tiefgefroren aufbewahrt, in kleinen Mengen an gefährdete Fohlen verfüttert werden. Ferner ist an eine Stutenimpfung zur Stimulierung und Verlängerung der Antikörpersekretion über Kolostrum und Milch, ähnlich wie bei anderen Spezies (Bachmann et al., 1982), zu denken. Kommerziell erhältliche Rotavirusvakzinen für eine Stutenimpfung existieren jedoch noch nicht.

\section{Literatur}

Bachmann, P. A. (1979): Rotavirusnachweis in Faeces: Erfahrungen mit dem Enzyme-linked Immunosorbent Assay (ELISA). Zbl. Vet. Med. B 26, 836-842.

Bachmann, P. A., und Hess, R. G. (1982): Comparative aspects of pathogenesis and immunity in animals. In: D. A. J. Tyrrell and A. S. Kapikian (Editors) Virus Infections of the Gastrointestinal Tract. Marcel Dekker, New York, NY, pp. 361-397.

Bachmann, P. A., Eichborn, W., Hess, R. G. (1982): Aktive Mutterschutzimpfung: passive Immunisierung von Neugeborenen. Tierärztl. Umsch. 37, 684-703.

Conner, M. E., and Darlington, R. W. (1980): Rotavirus infection in foals. Am. J. vet. Res. 41, 1699-1703.

Conner, M. E., Gillespie, J. H., Schiff, E. I., and Frey, M. S. (1983): Detection of rotavirus in horses with and without diarrhea by electron microscopy and Rotazyme test. Cornell Vet. 73, 280-287.

Durbam, P. J. K., Stevenson, B. J., and Farqubarson, B. C. (1979): Rotavirus and coronavirus associated diarrhoea in domestic animals. New Zeal. vet. J. 27, 30-32.

Eichborn, W., Werbabn, H., Jacobi, R., and Bachmann, P. A. (1985): Occurrence, isolation and characterization of equine rotaviruses. $\mathrm{Zbl}$. Vet. Med. B, in Druck.

Eugster, A. K., Whitford, H. W., and Mehr, L. E. (1978): Concurrent rotavirus and salmonella infections in foals. J. Am. Vet. med. Ass. 173, $857-858$.

Flewett, T. H., Bryden, A. S., and Davies, H. (1975): Virus diarrhoea in foals and other animals. Vet. Rec. 96, 477.

Gillespie, J. H., Kalica, A. R., Conner, M. E., Schiff, E., Barr, M., and Frey, M. (1984): The isolation, propagation and characterization of tissuecultured equine rotaviruses. Vet. Microbiol. 9, 1-14.

Hoshino, Y., Wyatt, R. G., Greenberg, H. B., Kalica, A. R., Flores, J., Kapikian, A. Z. (1983): Isolation and characterization of an equine rotavirus. J. clin. Microbiol. 18, 585-591.

Hoshino, Y., Wyatt, R. G., Greenberg, H. B., Kalica, A. R., Flores, J., Kapikian, A. Z. (1983): Isolation, propagation and characterization of a second equine rotavirus serotype. Infect. Immun. 41, 1031-1037.

Imagawa, H., Kirasawa, K., and Aikyama, Y. (1982): A seroepizootiological survey on rotavirus infection in foals. Jap. J. vet. Sci. 44, 819-821.

Rodger, S. M., Holmes, I. H., and Studdert, M. J. (1980): Characteristics of the genome of equine rotaviruses. Vet. Microbiol. 5, 243-248. 
Sattar, E. (1983): Personal Communication.

Smith, M., and Tzipori, S. (1979): Gel electrophoresis of rotavirus RNA derived from six different animal species. Austr. J. exp. Biol. Med. 57, $583-585$.

Snodgrass, D. R., and Herring, J. A. (1977): The action of disinfectants on lamb rotavirus. Vet. Rec. 101, 81 .

Strickland, K. L., Lenihan, P., O'Connor, M. G., and Condon, J. C. (1982): Diarrhea in foals associated with rotavirus. Vet. Rec. 111, 421.

Studdert, M. J., Mason, R. W., and Patten, B. E. (1978): Rotavirus diarrhoea of foals. Austr. vet. J. 54, 363-364.

Thouless, M. E., Bryden, A. S., Flewett, T. H., Woode, G. N., Bridger, J. C., Snodgrass, D. R., and Herring, J. A. (1977): Serological relationships between rotaviruses from different species as studied by complement fixation and neutralization. Arch. Virol. 53, 287-294.

Tzipori, S., and Walker, M. (1978): Isolation of rotavirus from foals with diarrhoeas. Austr. J. exp. Biol. Med. 56, 453-457.

Tzipori, G. R., Makin, T. J., Smith, M. L., (1980): The clinical response of gnotobiotic calves, pigs, and lambs to inoculation with human, calf, pig, and foal rotavirus isolates. Austral. J. exp. Biol. Med. 58, 309-318.
Tzipori, S. R., Makin, T. J., Smith, M. L., and Krautil, F. (1982): Enteritis in foals induced by rotavirus and enterotoxigenic Escherichia coli. Austral. vet. J. 58, 20-23.

Woode, G. N., Bew, M. E., Dennis, M. J. (1978): Studies on cross protection induced in calves by rotaviruses of calves, children, and foals. Vet. Rec. $103,32-34$.

Prof. Dr. P. A. Bachmann Institut für Med. Mikrobiologie Infektions. und Seuchenmedizin Ludreig-Maximilians-Universität

Tierärztliche Fakultät

Veterinärstraße 13

D-8000 München 22 